\title{
Comparative Effectiveness of Three Anxiolytics for Acute Respiratory Infections: Antibiotics, C-Reactive Protein Point-of-Care Testing, and Improved Communication
}

\author{
Jeffrey A. Linder, MD, MPH, FACP \\ KEY WORDS: respiratory tract infections; bronchitis; anti-bacterial agents; \\ physician-patient relations; health communication. \\ J Gen Intern Med 30(4):387-9 \\ DOI: $10.1007 /$ s11606-015-3181-1 \\ (C) Society of General Internal Medicine 2015
}

Division of General Medicine and Primary Care, Brigham and Women's Hospital and Harvard Medical School, Boston, MA, USA

A cute respiratory infections (ARIs) are a major burden on healthcare systems. ARIs - including non-specific upper respiratory infections (the common cold), otitis media, sinusitis, pharyngitis, acute bronchitis, influenza, and pneumonia - are the number-one symptomatic reason for seeking medical care. In the United States, ARIs account for about $10 \%$ of all ambulatory visits.

Among ARIs, the diagnosis and management of acute bronchitis should be particularly straightforward. Acute bronchitis is a cough-predominant respiratory infection of less than 3 weeks' duration in a patient without chronic cardiopulmonary disease who has normal vital signs and a normal lung examination. Forty years of randomized controlled trials, as well as more recent guidelines and performance measures, indicate that antibiotics are not beneficial for acute bronchitis and that the right antibiotic prescribing rate is zero.

Despite clear evidence, guidelines, and measures indicating that physicians should avoid prescribing antibiotics for acute bronchitis, doctors and patients remain anxious about withholding them. The Centers for Disease Control and Prevention and other organizations have implemented broad educational efforts, but the antibiotic prescribing rate for acute bronchitis in the United States is $70 \%$, and actually increased between 1996 and $2010 .^{1}$

There are many possible reasons that doctors continue to prescribe antibiotics for acute bronchitis despite the clear contravening evidence. Physicians and patients are confused by differences among "chest colds," "chest infections," "acute bronchitis," "lower respiratory tract infections," and "pneumonia." 2 Even if diagnostic definitions were perfect, in the real world, physicians are faced with uncertainty. ${ }^{3}$ Where does a cold stop and acute bronchitis start? Am I really sure my patient does not have pneumonia? Sinusitis? Undetected, underlying chronic lung disease?

Published online January 22, 2015
Acute bronchitis is anxiety-provoking for both doctors and patients, in part because it has a long course. Patients and physicians are anxious about an illness that, on average, resolves over the course of 3 weeks. ${ }^{4}$ Physicians are also anxious about keeping patients satisfied, and feel they have to do something in an effort to help patients feel better. Merely providing reassurance feels inadequate, and physicians have a difficult time conveying reassurance without trivializing patients' concerns. ${ }^{5}$ Finally, a few patients with acute bronchitis have complications, and a very small proportion of patients initially diagnosed with acute bronchitis will develop pneumonia. $^{6}$

To address this anxiety and reduce inappropriate antibiotic prescribing, the GRACE INTRO investigators have conducted a pragmatic multinational implementation tour de force. The investigators performed a 6-country controlled factorial cluster randomized trial of C-reactive protein (CRP) testing in comparison to communication training and the use of a patient booklet for patients with ARIs, most of whom probably had acute bronchitis. ${ }^{7}$ They evaluated CRP testing, given that prior studies had revealed that patients and physicians expressed a strong interest in testing that would reduce uncertainty and would clarify the need for antibiotics. The investigators evaluated communication through the use of an educational booklet, which had been found effective in prior studies. However, beyond measuring whether the interventions worked, they conducted a suite of adjunctive quantitative and qualitative studies in order to understand why the interventions worked and whether they were likely to continue working after the trial.

The trial included 228 practices, 372 physicians, and 4,264 patients, and found that CRP testing and communication training interventions were effective in reducing antibiotic prescribing. The antibiotic prescribing rate decreased in the CRP group from $48 \%$ to $33 \%$ and decreased in the communication and booklet group from $45 \%$ to $36 \%$. Each intervention had a downside: patients in the CRP had a low, but slightly higher rate of hospitalization; patients in the communication group reported 1 additional day of rating their symptoms "moderately bad or worse," perhaps because the patients had newly reset expectations about the duration of illness.

Some advantages of the communication and booklet intervention began to emerge in quantitative surveys of physicians. 
Physicians more reliably implemented techniques taught in communication training, and gave the booklet to $84 \%$ of patients. ${ }^{8}$ Physicians used CRP testing more selectively, for $38 \%$ of patients. Beyond the reliability of implementation, physicians in the communication group gained confidence in their ability to reduce antibiotic prescribing, and physicians in the CRP group lost confidence.

In quantitative surveys of patients, those in the communication practices expressed greater satisfaction and enablement - a concept related to self-efficacy, in which patients feel empowered to understand and cope with their health and illness - especially if they had received the booklet. ${ }^{8}$ Communication group patients had lesser belief in the necessity of antibiotics and greater belief in the harms of antibiotics. In contrast, patients in the CRP group had lower enablement scores. In fact, actually receiving the CRP test was associated with even lower scores of satisfaction and enablement.

In a qualitative study of patients' views of the intervention, patients liked both interventions and, consistent with many other studies, were satisfied with not receiving antibiotics. ${ }^{9}$ Patients in both groups planned to wait longer before seeking care in the future, but some in the CRP group expressed a desire to return for CRP testing the next time they were sick.

To this suite of studies, the GRACE INTRO investigators have added an article in the current issue of JGIM, a qualitative study from 66 semi-structured interviews of physicians who participated in one of the interventions. ${ }^{10}$ Communication training and the contents of the booklet gave participating physicians greater confidence in addressing patient expectations and reduced anxiety about not prescribing antibiotics. In particular, physicians felt the booklet gave patients a consistent backup source of information and provided a "safety netting" function, alerting patients to important red flags for which they should re-consult. Physicians felt CRP testing was most helpful in situations of uncertainty, but that it was difficult to use. Physicians, like patients, expressed concern that CRP testing could lead to more consultations in the future. There were no consistent differences in attitudes among physicians from the 6 countries, and there is little reason to think that attitudes would be different in the United States.

CRP testing has appeal and is effective in reducing antibiotic prescribing, ${ }^{11}$ but physicians do not persist in using it. One study of CRP testing in Norway was terminated due to a lack of difference in antibiotic prescribing rates and lack of interest from participating physicians. ${ }^{12}$ In a precursor to the GRACE INTRO study, Cals and colleagues followed patients who had attended practices randomized to CRP testing or communication training over the ensuing 3.5 years. ${ }^{13}$ Despite physician and patient concerns that CRP testing would lead to increased visits, both interventions were associated with a nonsignificant, roughly $30 \%$ decrease in the number of future respiratory infection visits and telephone calls. CRP testing was only used in $4 \%$ of subsequent respiratory visits, and placement in the CRP testing group was not associated with a persistent reduction in antibiotic prescribing. In contrast, communication training was associated with a persistently lower antibiotic prescribing rate.

Backing up a step, neither of the interventions in the GRACE INTRO study directly address the burden ARIs place on healthcare systems. What if there were solutions that could address patient and physician anxiety without an office visit at all? Patients want easy connection to their primary care physician, reassurance, the feeling that the system will respond if they get worse or need help navigating aspects of the sick role (e.g., excuse-from-work notes). Patients might be more conveniently triaged using online symptom checkers, phone calls, or asynchronous electronic visits. Modern electronic health records and communication technology, including messaging and video visits, could allow patients to connect to their primary care physicians' office throughout an episode of illness. Patient-reported outcomes could allow ongoing education and the same safety-netting function as the red-flag section of the GRACE INTRO informational booklet.

Patients and physicians are understandably anxious about the evaluation and management of acute bronchitis, but the comparative effectiveness of antibiotics, CRP testing, and improved communication seems clear. Antibiotics are ineffective and increase costs, increase the prevalence of antibioticresistant bacteria, and lead to adverse drug events. Point-ofcare testing with CRP is attractive on first consideration and is associated with a reduction in rates of antibiotic prescribing. However, implementation of CRP testing may be associated with increased hospitalizations, reduced physician confidence, and decreased patient satisfaction and enablement. CRP testing raises concerns about increased future healthcare usage, and physicians do not persist in using it.

Although not as sexy as a drug or as "exciting"-according to one study physician - as a new test, the best cure for ARIrelated anxiety appears to be improved communication, facilitated by the use of well-designed patient information materials. Physicians should talk with their patients, understand their concerns and expectations, perform a careful history and physical examination, and relay information about their decision-making and the illness, particularly regarding the usual 3-week course of acute bronchitis. Communication is the ARI anxiolytic that is generally free from adverse effects, improves physician and patient self-efficacy, is low-cost and effective, and is persistently implemented. Even better, a focus on improving communication has the potential to unburden primary care practices and should improve convenience and satisfaction for our patients.

Acknowledgments: Funding Support: Dr. Linder has been supported by grants from the National Institutes of Health (RC4 AG039115), the National Institute of Allergy and Infectious Diseases (R21 AI097759), and the Agency for Healthcare Research and Quality (R18 HSO18419).

Conflicts of Interest: Dr. Linder receives funding from Astellas Pharma, Inc. for research unrelated to antibiotic prescribing or respiratory infections. 
Corresponding Author: Jeffrey A. Linder, MD, MPH, FACP; Division of General Medicine and Primary Care Brigham and Women's Hospital and Harvard Medical School, Boston, MA, USA (e-mail: jlinder@partners.org).

\section{REFERENCES}

1. Barnett ML, Linder JA. Antibiotic prescribing for adults with acute bronchitis in the United States, 1996-2010. JAMA. 2014;311(19):2020-2022.

2. Woodhead M, Blasi F, Ewig S, et al. Guidelines for the management of adult lower respiratory tract infections-full version. Clin Microbiol Infect. 2011;17(Suppl 6):E1-E59.

3. Whaley LE, Businger AC, Dempsey PP, Linder JA. Visit complexity, diagnostic uncertainty, and antibiotic prescribing for acute cough in primary care: a retrospective study. BMC Fam Pract. 2013;14(1):120.

4. Ebell MH, Lundgren J, Youngpairoj S. How long does a cough last? Comparing patients' expectations with data from a systematic review of the literature. Ann Fam Med. 2013;11(1):5-13.

5. Cabral C, Ingram J, Hay AD, Horwood J. "They just say everything's a virus"-parent's judgment of the credibility of clinician communication in primary care consultations for respiratory tract infections in children: a qualitative study. Patient Educ Couns. 2014;95(2):248-253.

6. Meropol SB, Localio AR, Metlay JP. Risks and benefits associated with antibiotic use for acute respiratory infections: a cohort study. Ann Fam Med. 2013;11(2):165-172.
7. Little $\mathbf{P}$, Stuart B, Francis $\mathbf{N}$, et al. Effects of internet-based training on antibiotic prescribing rates for acute respiratory-tract infections: a multinational, cluster, randomised, factorial, controlled trial. Lancet. 2013;382(9899): 1175-1182.

8. Yardley L, Douglas E, Anthierens S, et al. Evaluation of a web-based intervention to reduce antibiotic prescribing for LRTI in six European countries: quantitative process analysis of the GRACE/INTRO randomised controlled trial. Implement Sci. 2013;8:134.

9. Tonkin-Crine S, Anthierens S, Francis NA, et al. Exploring patients' views of primary care consultations with contrasting interventions for acute cough: a six-country European qualitative study. NPJ Prim Care Respir Med. 2014;24:14026.

10. Anthierens S, Tonkin-Crine S, Cals Jw, et al. Clinicians' views and experiences of interventions to enhance the quality of antibiotic prescribing for acute respiratory tract infections. J Gen Intern Med. 2015; (SPI 3076).

11. Aabenhus R, Jensen JU, Jorgensen KJ, Hrobjartsson A, Bjerrum L. Biomarkers as point-of-care tests to guide prescription of antibiotics in patients with acute respiratory infections in primary care. Cochrane Database Syst Rev. 2014;11:CD010130.

12. Melbye H, Aaraas I, Fleten N, Kolstrup N, Mikalsen JI. The value of Creactive protein testing in suspected lower respiratory tract infections. A study from general practice on the effect of a rapid test on antibiotic research and course of the disease in adults. Tidsskr Nor Laegeforen. 1995;115(13):1610-1615.

13. Cals JW, de Bock L, Beckers PJ, et al. Enhanced communication skills and C-reactive protein point-of-care testing for respiratory tract infection 3.5-year follow-up of a cluster randomized trial. Ann Fam Med. 2013;11(2): 157-164. 